\title{
Translational research into gut microbiota: new horizons on obesity treatment: updated 2014
}

Daniela M. Tsukumo', Bruno M. Carvalho', Marco A. Carvalho-Filho', Mário J. A. Saad'

1 Department of Internal Medicine, University of Campinas (Unicamp), Campinas, SP, Brazil

\author{
Correspondence to: \\ Mário J. A. Saad \\ Departamento de Clínica Médica, \\ Faculdade de Ciências Médicas, \\ Universidade Estadual de Campinas \\ Cidade Universitária Zeferino Vaz \\ 13081-970 - Campinas, SP, Brazil \\ msaad@fcm.unicamp.br \\ Received on Jan/1/2014 \\ Accepted on June/29/2014 \\ DOI: 10.1590/2359-3997000000029
}

\begin{abstract}
Obesity is currently a pandemic of worldwide proportions affecting millions of people. Recent studies have proposed the hypothesis that mechanisms not directly related to the human genome could be involved in the genesis of obesity, due to the fact that, when a population undergoes the same nutritional stress, not all individuals present weight gain related to the diet or become hyperglycemic. The human intestine is colonized by millions of bacteria which form the intestinal flora, known as gut flora. Studies show that lean and overweight human may present a difference in the composition of their intestinal flora; these studies suggest that the intestinal flora could be involved in the development of obesity. Several mechanisms explain the correlation between intestinal flora and obesity. The intestinal flora would increase the energetic extraction of non-digestible polysaccharides. In addition, the lipopolysaccharide from intestinal flora bacteria could trigger a chronic sub-clinical inflammatory process, leading to obesity and diabetes. Another mechanism through which the intestinal flora could lead to obesity would be through the regulation of genes of the host involved in energy storage and expenditure. In the past five years data coming from different sources established causal effects between intestinal microbiota and obesity/insulin resistance, and it is clear that this area will open new avenues of therapeutic to obesity, insulin resistance and DM2. Arch Endocrinol Metab. 2015;59(2):154-60

Keywords

Obesity; gut flora; energy extraction; lipopolysaccharide
\end{abstract}

\section{INTRODUCTION}

I $\mathrm{n}$ recent years, the prevalence of obesity has increased rapidly throughout the World $(1,2)$. This epidemic is related to the intake of high calorie foods associated with sedentary lifestyles (3). The imbalance between energy expenditure, caloric intake and energy storage, is known to contribute to the development of obesity (4). Recent studies have suggested that mechanisms not directly related to the human genome could be involved in the genesis of obesity, due to the fact that, when a determined population undergoes similar nutritional stress, not all individuals become hyperglycemic or present diet-related weight gain (5-7).

The human intestine is colonized by millions of bacteria, primarily anaerobic bacteria, comprising approximately 1,000 species (8). The intestinal flora genome has approximately 100 times more genes than the human genome $(5,9)$. Normal intestinal flora presents different functions that contribute to the functioning of our organism, therefore this flora can be considered a microbial organ $(10,11)$.
Recently several studies have suggested a direct role of the intestinal flora in the development of obesity, as the intestinal flora could alter energetic and fat storage (4). In this article, we review the potential role of the intestinal flora in the development of obesity.

\section{NORMAL GUT MICROBIOTA}

A large number of bacteria inhabit the gastrointestinal tract and the genome of these commensal bacteria which constitute the intestinal flora is greater than the human genome $(12,13)$.

The stomach and the small intestine are not colonized by a large number of bacteria, as the pancreatic secretion and bile acids prevent bacterial proliferation (13). The colon however, presents approximately $10^{12}$ microorganisms/g of intestinal content (14) (Figure 1). Recent studies have highlighted that over $90 \%$ of these bacteria are Bacteroides and Firmicutes (4), furthermore, over $90 \%$ of these bacteria are compulsory anaerobes. The predominant bacterial species are: 
Bacteroidetes, Eubacterium, Bifidobacterium, Fusobacterium, Peptostreptococcus, amongst others.

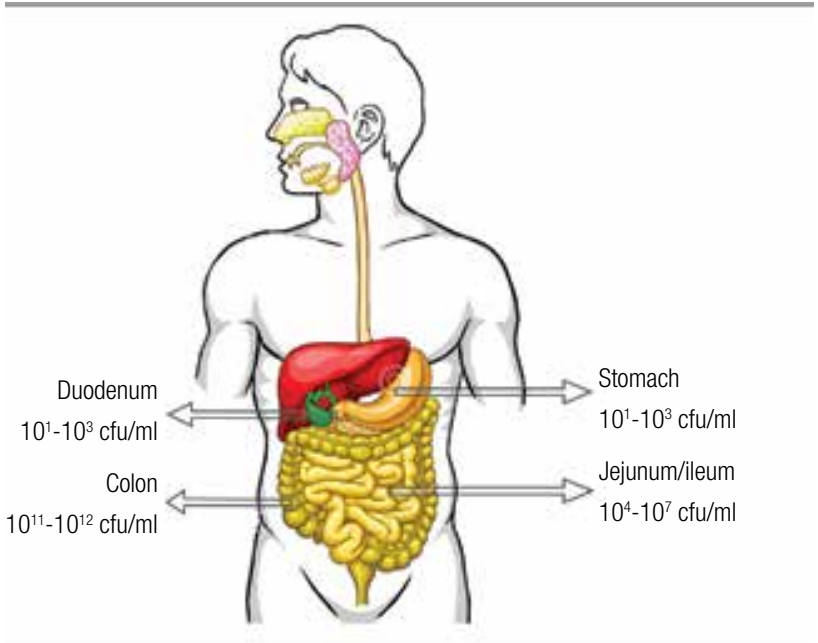

Figure 1. Relative concentrations of bacteria at various locations within the gut. Cfu: colony-forming unit.

Commensal bacteria have a fundamental role in functioning of the intestine (15). These bacteria are known to have several functions, such as cellulose digestion (14) and also form a protective barrier that participates in the development of the systemic and mucosal immune system (15). The gut flora is constituted by multiple non-pathogenic bacteria, and also contains possible pathogenic microorganisms (15).

The composition of the intestinal flora is influenced by several factors and appears to be determined during the first year of life (4). Factors that contribute to the establishment of the this flora include forms of childbirth delivery, diet of the newborn, use of medicine and hygiene level, as the intestine is sterile at birth $(13,16,17)$. The establishment of the intestinal flora begins at delivery when the newborn comes into contact with the bacteria present in the environment, in maternal feces and bacteria of the vaginal flora (18). The intestinal flora is probably continued to be built up for several months after birth, when a stable and unique flora is established (19-21). The form of delivery may influence the initial intestinal flora composition. Cesarean section delivered newborns may present a 30 day delay in the colonization of the intestine with beneficial bacteria such as Bifidobacterium and Lactobacillus in comparison to vaginal delivered newborns $(16,22)$.

Subsequently, the composition of the intestinal flora undergoes substantial modifications and the diet of the infant begins to affect the composition and sequence of intestinal colonization. Breast fed newborns present differences compared to formula-fed newborns in the constitution of their intestinal flora (23). The intestinal flora of breastfed newborns limits the over-growth of potentially pathogenic bacteria such as Escherichia coli and Clostridium perfringens, through the production of acetate and lactate (15). The main component of the flora of these newborns is Bifidobacterium. Gestational age also affects the formation of the intestinal flora, preterm newborns in the neonatal intensive care unit have been observed to have a lower number of bacteria species, and the strains tended to be more virulent $(24,25)$.

Changes in the composition of intestinal microbiota occurs along the human intestinal tract and throughout the life of an individual, the composition of the flora however is quite stable during most of the individual's life (15). The change to an adult diet, factors of the intestinal flora itself, changes in the intestinal environment, amongst other factors, contribute to the transformation of the intestinal flora into an adult type of flora $(26,27)$.

Age appears to be another factor involved in the modification of intestinal flora. The elderly present a lower number of anaerobic bacteria, and on the other hand, a higher number of enterobacteria $(28,29)$. Aging brings on a significant increase in the potentially pathogenic bacteria such as enterobacterias and Clostridium, and a decrease in the Bifidobacterial species, which contribute to the protection of the intestinal tract $(30,31)$.

The intestinal flora may vary considerably from one individual to another. As observed in studies with adults with a varying degree of kinship, genotype appears to be the most important factor in defining the composition of the flora. Additional factors such as diet, life expectancy and age would have a less relevant role in the composition of an individual's flora $(28,32)$.

The intestinal flora plays an essential role in maintaining normal gastrointestinal tract function; this includes the digestion of nutrients and immune response of the gastrointestinal tract. Animal studies using germ-free mice revealed that these animals presented a decrease in the activity of digestive enzymes, of the mucosa-associated lymphoid tissue and reduction of motility, muscle wall thickness and vascularization. These animals also presented a decrease in serum immunoglobulin levels and were more prone to infections $(12,13)$. 


\section{GUT MICROBIOTA AND OBESITY}

\section{Extraction of additional calories from ingested food}

Recent studies have shown that the bacteria present in the intestinal flora is capable of altering energy regulation and harvesting energy from nutrients, suggesting that the flora can play a role in the development of obesity (4). Moreover, in hyperlipidemic diet-induced obesity, the lipopolysaccharide from intestinal bacteria could represent a triggering factor for the inflammation present in these obesity models (Figure 2).

An animal study using germ-free mice observed that these mice, despite ingesting greater amounts of food than conventionally raised mice, presented a lower amount of body fat (8). Subsequently, a study by Turnbaugh and cols., highlighted that germ-free mice colonized with the gut flora from obese mice, presented a higher increase in body fat than when they were colonized with the flora from lean animals (33). This same study showed that obese $o b / o b$ mice (leptin-deficient mice) had a reduced number of Bacteroidetes and a proportional increase in Firmicutes, when compared to lean mice $(\mathrm{ob} /+$ and $+/+)$ from the same litter. Furthermore, the gut flora of obese mice was observed to

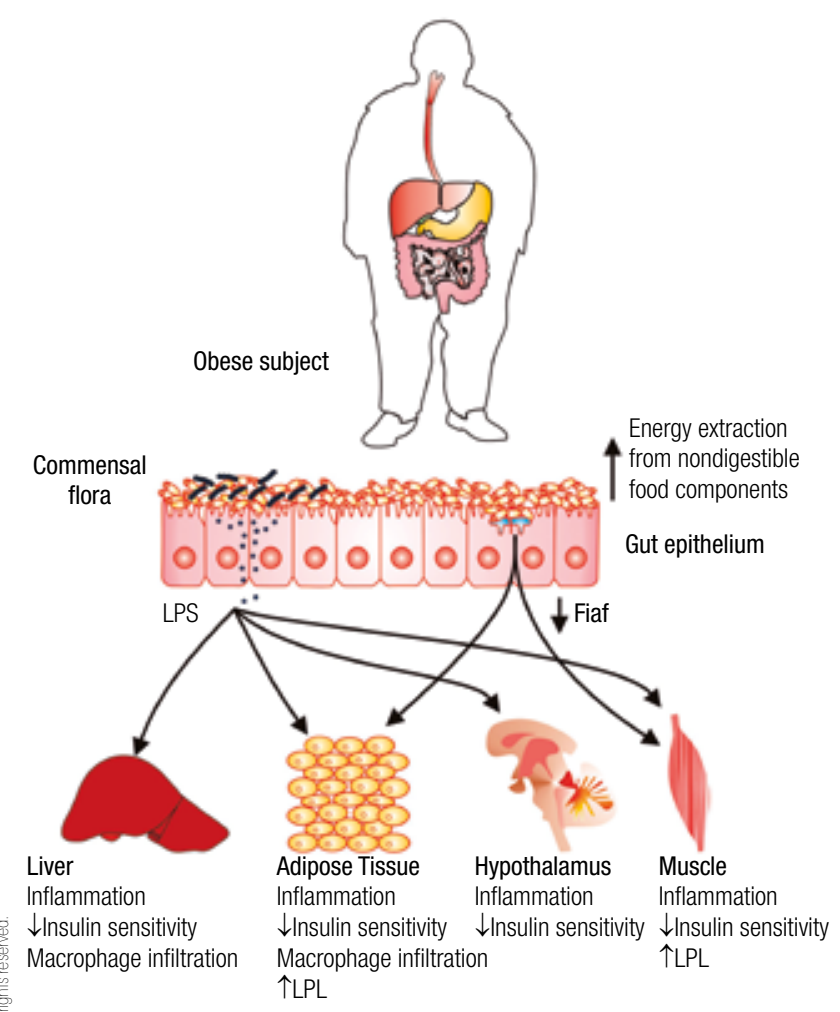

Figure 2. Schematic view of the possible mechanisms linking gut flora to obesity. FIAF: fasting-induced adipose factor; LPL: lipoprotein lipase; LPS: lipopolysaccharide. present an abundance of genes coding for enzymes capable of digesting polysaccharides from diet not usually digestible by human. In addition, these investigators proposed the hypothesis that the flora of obese mice favored a greater capacity of extracting calories from food, as the feces of these mice were observed to have less calories and a greater amount of fermentation end-products.

Ley and cols. highlighted the gut flora imbalance of $o b / o b$ mice compared to that of lean mice in another study, observing a relative increase of $50 \%$ of Firmicutes and a decrease of $50 \%$ of Bacteroidetes in $o b / o b$ mice (34) (Figure 3). These same investigators demonstrated that obese individuals presented an intestinal flora with a lower proportion of Bacteroidetes than lean persons (35). Weight loss over a one-year period modifies the flora of obese individuals, leveling the proportion of Firmicutes close to those observed in lean persons.
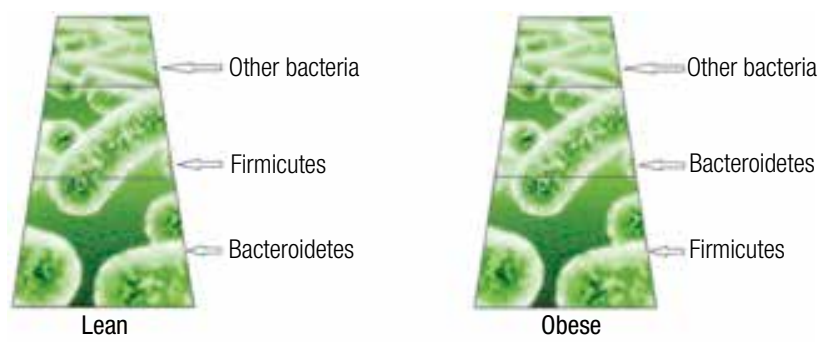

Figure 3. Relative proportion of Firmicutes and Bacteroidetes in lean and obese mice.

The correlation between intestinal flora and obesity requires further studies in order to elucidate several questions. It is not known yet whether, as proposed by studies on transplantation of flora, a significant weight gain could occur, over a short period of time, simply by a small increase in the extraction of energy (36). A diet rich in non-digestible fibers has been observed to reduce the severity of diabetes, reduce weight and the amount of fat tissue $(37,38)$.

Additionally other important questions remain unanswered, for example, in which manner do weight differences alter the balance of the intestinal flora is not yet clear. Furthermore, the manner by which the change in flora could be triggered by the conditions of the host organism has not yet been fully elucidated (39). The intestinal flora of obese individuals has been suggested to undergo changes which would increase the extraction of calories from nutrients; this however would seemingly be more adaptive if it were to occur in individuals who presented weight loss, interestingly however it is precisely the intestinal flora of obese human, 
who already have high calorie reserves, that has been observed to be more efficient (35).

\section{Induction of subclinical inflammation}

A short time ago, several studies highlighted a correlation between type- 2 diabetes and obesity which a state of subclinical chronic inflammation in several tissues such as the adipose tissue, liver and hypothalamus (4044). Based on this observation, a new hypothesis has been proposed correlating intestinal flora and obesity (Figure 2). The inflammation that leads to diabetes and obesity in high-fat diet animal models has been proposed to be triggered by the lipopolysaccharide (LPS) of gram-negative bacteria which compose the intestinal flora (45). These investigators connected an altered microbiota composition, brought about by a high-fat diet, to an increase in the proportion of gram-negative bacteria compared to gram-positive bacteria, with a decrease in Bacteroides, Eubacterium rectal-Clostridium coccoides group and bifidobacteria. In this study, the imbalance of the intestinal microbiota associated to a high-fat diet was correlated to insulin resistance, diabetes, and increase in body weight gain and body fat, increase in serum LPS and triglycerides levels in the liver. Additionally, another study observed a decrease in hepatic steatosis and LPS expression after treating rats with polymixin $\mathrm{B}$ an antibiotic specific for gram-negative bacteria (46).

In human, individuals without type 2 diabetes presented lower levels of serum lipopolysaccaride than patients with type 2 diabetes, paired by age (47). These data corroborate the hypothesis that the development of obesity and type 2 diabetes can be correlated to the lipopolysaccharide from intestinal flora bacteria.

Another animal study using $o b / o b$ mice, demonstrated that the modulation of the gut flora using antibiotics (ampicillin and norfloxacin) can reduce hepatic steatosis and improve glucose tolerance (48). Moreover, mice treated with a high-fat diet, were observed to present a reduction in intestinal permeability and in serum LPS levels, in addition to a decrease in the inflammation of adipose tissue and macrophage infiltration, after the modification of gut microbiota using antibiotics (49-53).

\section{Regulation of genes in the host involved in energy storage and expenditure}

In a recent study, Backhed and cols. highlighted that control animals compared to germ-free mice, presented
$40 \%$ more body fat and approximately $50 \%$ more gonadal fat (8). After two weeks, germ-free mice who had received the gut flora of control mice were observed to present an increase of $60 \%$ of body fat. Two proteins carbohydrate response element-binding protein (ChREBP) and liver sterol response element-binding protein type-1 (SREBP-1) were demonstrated to be involved in the absorption of monosaccharides in the intestine and hepatic lipogenesis induced by the gut flora (8).

The authors suggested that the intestinal flora interferes in the energy metabolism through several mechanisms, among which is included the absorption of short chain fatty acids and monosaccharides, which are subsequently transformed into fat in the liver. Furthermore, the intestinal flora would enable the absorption of polysaccharides which are not normally digestible and enable the regulation of genes in the host which are involved in the fat deposit in adipocytes (8). Backhed and cols., investigated the role of lacking fasting-induced adipose factor (Fiaf) or angiopoietin-like protein 4 in the modulation of microbiota induced-weight control and showed that the gut flora suppressed intestinal Fiaf (Figure 2), leading to increased lipoprotein lipase activity in adipocytes, with a consequent increase of fat (8).

Backhed and cols. (54) suggested that germ-free mice were resistant to obesity, even when they consumed a diet rich in fat and sugar, through mechanisms that involved an increase in the metabolism of fatty acids. They demonstrated an increase in the activity of the AMPK (adenosine monophosphate-activated protein kinase) enzyme, which plays a central role in the regulation of homeostasis, as a sensor of the status of cell energy in the cell. In addition, an increase in Fiaf levels triggers the production of peroxisome proliferator-activated receptor gamma coactivator, increasing the expression of genes that regulate the oxidation of fatty acids in the mitochondria. In this way, the intestinal flora appears to influence the energy balance not only by enabling the extraction of energy from nutrients, but also by influencing the expression of genes with regulate energy expenditure and storage (54).

\section{CONCLUSION IN 2009}

Studies showing that lean and obese humans present differences in intestinal flora, suggest that this flora could be involved in the development of obesity. Sever- 
al mechanisms explain the correlation between intestinal flora and obesity. The intestinal flora would increase the energy extraction from non-digestible polysaccharides. Moreover, the lipopolysaccharide from bacteria flora would trigger a state of subclinical chronic inflammation, leading to obesity and diabetes. Another mechanism through which the intestinal flora would lead to obesity would be through the regulation of genes in the host, involved in energy storage and expenditure. However, further studies are necessary to elucidate several issues related to the association between intestinal flora and obesity. It is not yet clear whether the change in the intestinal flora observed in obese individuals causes or is caused by obesity. In addition, it is not yet known whether a significant weight gain could occur due to a modest increase in calorie extraction.

The human intestine is colonized by millions of microorganisms and the modifications in the composition of the intestinal flora could constitute a novel type of treatment for obesity. Probiotics are live microorganisms that could promote changes in the composition of intestinal flora. Prebiotics stimulate the growth and activity of commensal bacteria as they are non-digestible oligosaccharides fermented by the intestinal flora. Antibiotics, probiotics and prebiotics could possibly have a role in the manipulation of intestinal flora to treat obesity in the future.

\section{CONCLUSION IN 2014}

Since the relationship between obesity and gut microbiota was established few years ago, the important question was whether this relationship is causal. In this regard, in the past five years, data coming from different sources have shown a causal relationship between gut microbiota and obesity $(4,5,55)$, supported by experiments including microbiota modulation and mainly transplantation. In addition, studies in mice and in humans have also shown that gut microbiota have a causal role in insulin resistance, which is the primary alteration observed in DM2. In this regard, an elegant study of Ridaura and cols. (55), demonstrated that microbiota transplantation from obese or lean human to germ free mice induced obesity or lean phenotypes, respectively. They also showed that housing mice with obese microbiota with mice with lean microbiota prevented the development of obese phenotypes in the former (55). These data indicated us clearly that microbiota can improve metabolic parameters or even prevent obesity, al- though the composition of diet will also be important $(11,14,36,39,55,56)$.

In addition to the causal role of intestinal microbiota on obesity and insulin resistance, alterations in gut microbiota have been reported in association with HIV disease progression (57), with olanzapine-induced metabolic dysfunction (58) and increasing the risk of cardiovascular diseases (59). Gut microbiota can also modulate diurnal secretion of glucocorticoids $(60)$, the immunity with important role in the regulation of Treg cells (61), and surprisingly brain activity (62). These alterations/modulations usually are induced by products of bacterial metabolism as short chain fatty acids, kinurenin which is a product of tryptophan catabolism by some bacteria, choline and phosphatidylcholine which will first produce trimethylamine (TMA) and then a proatherogenic compound trimethylamine- $\mathrm{N}$-oxide (TMAO) and many others not yet identified.

We should also mention that drugs used in clinical practice can have a direct effect on intestinal microbiota, or its metabolism may also be influenced by microbiota. Recently it was demonstrated that metformin can modulate intestinal microbiota, at least in C. elegans, suggesting that the therapeutic effect and also the side effects of this drug may also be mediated by microbiota (63). Another interesting study in this area is related to the inactivation of cardiac drug digoxin by gut bacteria (Eggerthella lenta), suggesting an important role of diet and microbioma in inducing digitalis intoxication (64).

Finally it is important to recognize that this area suffers an enormous progress in the past years, but the ideal microbiota profile to a specific person remains to be established. However we should be optimistic because this new knowledge may allowed us to establish new clinical trials with slow release encapsulated microbiota or probiotics in order to treat obesity/DM2 or even to introduce an important epidemic of leanness.

Note of Editor: this manuscript is the corrected and revised version, and invalidates a previous manuscript published in 2009 that was retracted.

Disclosure: no potential conflict of interest relevant to this article was reported.

\section{REFERENCES}

1. Hill JO, Wyatt HR, Reed GW, Peters JC. Obesity and the environment: where do we go from here? Science. 2003;299(5608):853-5.

2. Raoult D. Obesity pandemics and the modification of digestive bacterial flora. Eur J Clin Microbiol Infect Dis. 2008;27(8):631-4.

3. Hill JO. Understanding and addressing the epidemic of obesity: an energy balance perspective. Endocr Rev. 2006;27(7):750-61. 
4. DiBaise JK, Zhang H, Crowell MD, Krajmalnik-Brown R, Decker GA, Rittmann BE. Gut microbiota and its possible relationship with obesity. Mayo Clin Proc. 2008;83(4):460-9.

5. Cani PD, Delzenne NM. Gut microflora as a target for energy and metabolic homeostasis. Curr Opin Clin Nutr Metab Care. 2007;10(6):729-34.

6. Levin BE, Keesey RE. Defense of differing body weight set points in diet-induced obese and resistant rats. Am J Physiol. 1998;274(2 Pt 2):R412-9.

7. Tappy L. Metabolic consequences of overfeeding in humans. Curr Opin Clin Nutr Metab Care. 2004;7(6):623-8.

8. Backhed F, Ding H, Wang T, Hooper LV, Koh GY, Nagy A, et al. The gut microbiota as an environmental factor that regulates fat storage. Proc Natl Acad Sci U S A. 2004;101(44):15718-23.

9. Xu J, Gordon JI. Inaugural article: honor thy symbionts. Proc Natl Acad Sci U S A. 2003;100(18):10452-9.

10. Bocci $V$. The neglected organ: bacterial flora has a crucial immunostimulatory role. Perspect Biol Med. 1992;35(2):251-60.

11. Backhed F, Ley RE, Sonnenburg JL, Peterson DA, Gordon JI. Host-bacterial mutualism in the human intestine. Science. 2005;307(5717):1915-20.

12. Shanahan F.The host-microbe interface within the gut. Best Pract Res Clin Gastroenterol. 2002;16(6):915-31.

13. O'Hara AM, Shanahan F.The gut flora as a forgotten organ. EMBO Rep. 2006;7(7):688-93.

14. Mueller C, Macpherson AJ. Layers of mutualism with commensal bacteria protect us from intestinal inflammation. Gut. 2006;55(2):276-84.

15. Wolowczuk I, Verwaerde C, Viltart O, Delanoye A, Delacre M, Pot $B$, et al. Feeding our immune system: impact on metabolism. Clin Dev Immunol. 2008;2008:639803.

16. Gronlund MM, Lehtonen OP, Eerola E, Kero P. Fecal microflora in healthy infants born by different methods of delivery: permanent changes in intestinal flora after cesarean delivery. J Pediatr Gastroenterol Nutr. 1999;28(1):19-25.

17. Palmer C, Bik EM, DiGiulio DB, Relman DA, Brown PO. Development of the human infant intestinal microbiota. PLoS biology. 2007;5(7):e177.

18. Prelog M, Grif K, Decristoforo C, Wurzner R, Kiechl-Kohlendorfer U, Brunner A, et al. Tetracycline-resistant Escherichia coli strains are inherited from parents and persist in the infant's intestines in the absence of selective pressure. Eur J Pediatr. 2009;168(10):1181-7.

19. Favier CF, Vaughan EE, De Vos WM, Akkermans AD. Molecular monitoring of succession of bacterial communities in human neonates. Appl Environ Microbiol. 2002;68(1):219-26.

20. Midtvedt AC, Midtvedt T. Production of short chain fatty acids by the intestinal microflora during the first 2 years of human life. J Pediatr Gastroenterol Nutr. 1992;15(4):395-403.

21. Zoetendal EG, Akkermans AD, De Vos WM. Temperature gradient gel electrophoresis analysis of 16S rRNA from human fecal samples reveals stable and host-specific communities of active bacteria. Appl Environ Microbiol. 1998;64(10):3854-9.

22. Claud EC, WalkerWA. Bacterial colonization, probiotics, and necrotizing enterocolitis. J Clin Gastroenterol. 2008;42 Suppl 2:S46-52.

23. Mountzouris KC, McCartney AL, Gibson GR. Intestinal microflora of human infants and current trends for its nutritional modulation. Br J Nutr. 2002;87(5):405-20.

24. Kosloske AM. Epidemiology of necrotizing enterocolitis. Acta Paediatr Suppl. 1994;396:2-7.

25. Orrhage K, Nord CE. Factors controlling the bacterial colonization of the intestine in breastfed infants. Acta Paediatr Suppl. 1999;88(430):47-57.

26. Gorbach SL. Intestinal microflora. Gastroenterology. 1971;60(6):1110-29.
27. Mackie RI, Sghir A, Gaskins HR. Developmental microbial ecology of the neonatal gastrointestinal tract. Am J Clin Nutr. 1999;69(5):1035S-45S.

28. Hopkins MJ, Sharp R, Macfarlane GT. Age and disease related changes in intestinal bacterial populations assessed by cell culture, 16S rRNA abundance, and community cellular fatty acid profiles. Gut. 2001;48(2):198-205.

29. Hebuterne $X$. Gut changes attributed to ageing: effects on intestinal microflora. Curr Opin Clin Nutr Metab Care. 2003;6(1):49-54.

30. Gorbach SL, Nahas L, Lerner PI, Weinstein L. Studies of intestinal microflora. I. Effects of diet, age, and periodic sampling on numbers of fecal microorganisms in man. Gastroenterology. 1967;53(6):845-55.

31. Mitsuoka T. Recent trends in research on intestinal flora. Bifidobacteria Microflora. 1982;1:3-24.

32. Zoetendal EG, Akkermans ADL, Akkermans-van WM, de Visser JAGM, de Vos WM. The host genotype affects the bacterial community in the human gastrointestinal tract. Microb Ecol Health Dis. 2001;13(3):129-34.

33. Turnbaugh PJ, Ley RE, Mahowald MA, Magrini V, Mardis ER, Gordon JI. An obesity-associated gut microbiome with increased capacity for energy harvest. Nature. 2006;444(7122):1027-31.

34. Ley RE, Backhed F, Turnbaugh P, Lozupone CA, Knight RD, Gordon JI. Obesity alters gut microbial ecology. Proc Natl Acad Sci U S A. 2005;102(31):11070-5.

35. Ley RE, Turnbaugh PJ, Klein S, Gordon Jl. Microbial ecology: human gut microbes associated with obesity. Nature. 2006;444(7122):1022-3.

36. Cani PD, Delzenne NM, Amar J, Burcelin R. Role of gut microflora in the development of obesity and insulin resistance following high-fat diet feeding. Pathol Biol (Paris). 2008;56(5):305-9.

37. Cani PD, Joly E, Horsmans $Y$, Delzenne NM. Oligofructose promotes satiety in healthy human: a pilot study. Eur J Clin Nutr. 2006;60(5):567-72.

38. Cani PD, Neyrinck AM, Maton N, Delzenne NM. Oligofructose promotes satiety in rats fed a high-fat diet: involvement of glucagonlike Peptide-1. Obes Res. 2005;13(6):1000-7.

39. Bajzer M, Seeley RJ. Physiology: obesity and gut flora. Nature. 2006;444(7122):1009-10.

40. Wellen KE, Hotamisligil GS. Inflammation, stress, and diabetes. J Clin Invest. 2005;115(5):1111-9.

41. Caricilli AM, Nascimento PH, Pauli JR, Tsukumo DM, Velloso LA Carvalheira JB, et al. Inhibition of toll-like receptor 2 expression improves insulin sensitivity and signaling in muscle and white adipose tissue of mice fed a high-fat diet. $\mathrm{J}$ Endocrinol. 2008;199(3):399-406.

42. Carvalho-Filho MA, Ueno M, Hirabara SM, Seabra AB, Carvalheira JB, de Oliveira MG, et al. S-nitrosation of the insulin receptor, insulin receptor substrate 1 , and protein kinase B/Akt: a novel mechanism of insulin resistance. Diabetes. 2005;54(4):959-67.

43. Prada PO, Zecchin HG, Gasparetti AL, Torsoni MA, Ueno M, Hirata $A E$, et al. Western diet modulates insulin signaling, c-Jun $N$ terminal kinase activity, and insulin receptor substrate-1ser307 phosphorylation in a tissue-specific fashion. Endocrinology. 2005;146(3):1576-87.

44. Tsukumo DM, Carvalho-Filho MA, Carvalheira JB, Prada PO, Hirabara SM, Schenka AA, et al. Loss-of-function mutation in Tolllike receptor 4 prevents diet-induced obesity and insulin resistance. Diabetes. 2007;56(8):1986-98.

45. Cani PD, Amar J, Iglesias MA, Poggi M, Knauf C, Bastelica D, et al. Metabolic endotoxemia initiates obesity and insulin resistance. Diabetes. 2007;56(7):1761-72.

46. Pappo I, Becovier H, Berry EM, Freund HR. Polymyxin B reduces cecal flora, TNF production and hepatic steatosis during total parenteral nutrition in the rat. J Surg Res. 1991;51(2):106-12. 
47. Creely SJ, McTernan PG, Kusminski CM, Fisher M, Da Silva NF, Khanolkar $\mathrm{M}$, et al. Lipopolysaccharide activates an innate immune system response in human adipose tissue in obesity and type 2 diabetes. Am J Physiol Endocrinol Metab. 2007;292(3):E740-7.

48. Membrez M, Blancher F, Jaquet M, Bibiloni R, Cani PD, Burcelin RG, et al. Gut microbiota modulation with norfloxacin and ampicillin enhances glucose tolerance in mice. Faseb J. 2008;22(7):2416-26.

49. Cani PD, Bibiloni R, Knauf $C$, Waget A, Neyrinck AM, Delzenne $\mathrm{NM}$, et al. Changes in gut microbiota control metabolic endotoxemia-induced inflammation in high-fat diet-induced obesity and diabetes in mice. Diabetes. 2008;57(6):1470-81.

50. Caricilli AM, Picardi PK, de Abreu LL, Ueno M, Prada PO, Ropelle ER, et al. Gut microbiota is a key modulator of insulin resistance in TLR 2 knockout mice. PLoS biology. 2011;9(12):e1001212.

51. Caricilli AM, Saad MJ.The role of gut microbiota on insulin resistance. Nutrients. 2013;5(3):829-51.

52. Carvalho BM, Guadagnini D, Tsukumo DM, Schenka AA, LatufFilho $\mathrm{P}$, Vassallo J, et al. Modulation of gut microbiota by antibiotics improves insulin signalling in high-fat fed mice. Diabetologia. 2012;55(10):2823-34.

53. Carvalho BM, Saad MJ. Influence of gut microbiota on subclinical inflammation and insulin resistance. Mediators Inflamm. 2013;2013:986734.

54. Backhed F, Manchester JK, Semenkovich CF, Gordon JI. Mechanisms underlying the resistance to diet-induced obesity in germfree mice. Proc Natl Acad Sci U S A. 2007;104(3):979-84.

55. Ridaura VK, Faith JJ, Rey FE, Cheng J, Duncan $A E, K a u A L$, et al. Gut microbiota from twins discordant for obesity modulate metabolism in mice. Science. 341(6150):1241214.
56. Gibson GR, Roberfroid MB (eds.). Colonic Microbiota, Nutrition and Health. Springer Netherlands; 1999.

57. Vujkovic-Cvijin I, Dunham RM, Iwai S, Maher MC, Albright RG, Broadhurst MJ, et al. Dysbiosis of the gut microbiota is associated with hiv disease progression and tryptophan catabolism. Sci Trans Med. 2013;5(193):193ra91.

58. Davey KJ, Cotter PD, O'Sullivan O, Crispie F, Dinan TG, Cryan JF, et al. Antipsychotics and the gut microbiome: olanzapine-induced metabolic dysfunction is attenuated by antibiotic administration in the rat. TransI Psychiatry. 2013;3:e309.

59. Koeth RA, Wang Z, Levison BS, Buffa JA, Org E, Sheehy BT, et al. Intestinal microbiota metabolism of L-carnitine, a nutrient in red meat, promotes atherosclerosis. Nat Med. 2013;19(5):576-85.

60. Mukherji A, Kobiita A, YeT, Chambon P. Homeostasis in intestinal epithelium is orchestrated by the circadian clock and microbiota cues transduced by TLRs. Cell. 2013;153(4):812-27.

61. FurusawaY, ObataY, Fukuda S, EndoTA, Nakato G, Takahashi D, et al. Commensal microbe-derived butyrate induces the differentiation of colonic regulatory T cells. Nature. 2013;504(7480):446-50.

62. Tillisch K, Labus J, Kilpatrick L, Jiang Z, Stains J, Ebrat B, et al. Consumption of fermented milk product with probiotic modulates brain activity. Gastroenterology. 2013;144(7):1394-401, 401 e1-4.

63. Cabreiro F, Au C, Leung KY, Vergara-Irigaray N, Cocheme HM, Noori $\mathrm{T}$, et al. Metformin retards aging in $\mathrm{C}$. elegans by altering microbial folate and methionine metabolism. Cell. 2013;153(1):228-39.

64. Haiser HJ, Gootenberg DB, Chatman K, Sirasani G, Balskus EP, Turnbaugh PJ. Predicting and manipulating cardiac drug inactivation by the human gut bacterium Eggerthella lenta. Science. 2013;341(6143):295-8. 